\title{
A novel approach in Multi-hop networks technology with the ratio distribution of two Hyper-Erlang random variables
}

\author{
Therrar Kadri ${ }^{1}$, Khaled Smaili ${ }^{2}$, Seifedine Kadry ${ }^{3, *}$ \\ ${ }^{1}$ Department of Mathematics, Faculty of Sciences, Lebanese International University, Al-Khyara, Lebanon \\ ${ }^{2}$ Department of Applied Mathematics, Faculty of Sciences, Lebanese University, Zahle, Lebanon \\ ${ }^{3}$ School of Engineering, American University of the Middle East, Eguaila, Kuwait
}

(Received: 25 April 2015; Accepted: 10 September 2015)

\begin{abstract}
The distribution of ratio of two random variables has been studied by several authors especially when the two random variables are independent and come from the same family. In this paper, the exact distribution of the ratio of two independent Hyper-Erlang distribution is derived. However, closed expressions of the probability density, cumulative distribution function, reliability function, hazard function, moment generating function and the rth moment are found for this ratio distribution and proved to be a linear combination of the Generalized-F distribution. Finally, we will apply our results to real life application in analyzing the performance of wireless communication systems.
\end{abstract}

Keywords Ratio Distribution; Hyper-Erlang Distribution; Erlang Distribution; Generalized-F Distribution; Log-logistic Distribution; Probability Density Function; Survivor function; Moment Generating Function.

\section{AMS 2010 subject classifications 62E15, 60E10}

DOI: $10.19139 /$ soic.v3i4.143

\section{Introduction}

The distributions of ratio of random variables are of interest in many areas of the sciences, engineering, physics, number theory, order statistics, economics, biology, genetics, medicine, hydrology, psychology, classification, and ranking and selection, see [1, 2, 3, 4]. Examples include safety factor in engineering, mass to energy ratios in nuclear physics, target to control precipitation in meteorology, inventory ratios in economics and Mendelian inheritance ratios in genetics, see [1,2]. Also ratio distribution involving two Gaussian random variables are used in computing error and outage probabilities, see [5]. It has many applications especially in engineering concepts such as structures, deterioration of rocket motors, static fatigue of ceramic components, fatigue failure of aircraft structures and the aging of concrete pressure vessels, see [6, 7]. An important example of ratios of random variables is the stress strength model in the context of reliability. It describes the life of a component which has a random strength $Y$ and is subjected to random stress $X$. The component fails at the instant that the stress applied to it exceeds the strength and the component will function satisfactorily whenever $Y>X$. Thus, $\operatorname{Pr}(X<Y)$ is a measure of component reliability see [6, 7].

The ratio distribution $X / Y$ have been studied by several authors especially when $X$ and $Y$ are independent random variables and come from the same family. For a historical review, see the papers by Marsaglia [8] and Korhonen and Narula [9] for the normal family, Press [10] for Student's t family, Basu and Lochner [11] for the Weibull family, Hawkins and Han [12] for the non-central chi-squared family, Provost [13] for the gamma family,

*Correspondence to: Seifedine Kadry (Email: skadry@gmail.com).

ISSN 2310-5070 (online) ISSN 2311-004X (print)

Copyright (C) 2015 International Academic Press 
Pham-Gia [14] for the beta family, Nadarajah and Gupta [15] for the Logistic family, Nadarajah and Kotz [16] for the Frèchet family, Ali, Pal, and Woo [1] for the inverted gamma family, Nadarajah [17] for Laplace family, and [7] for the Generalized-F family.

The distribution $X / Y$, when $X$ and $Y$ are two independent Hyper-Erlang distributions, was not examined by any author. In this paper, we examine this distribution and find a closed expression of the probability density function (PDF). We showed that the PDF of this distribution is a linear combination of the Generalized-F distribution. As a consequence, the ratio distribution of two independent Hyper-Erlang distributions is obtained from the known Generalized-F distribution. Thus, the expressions of the cumulative distribution function (CDF), reliability function, hazard function, moment generating function and moments of order $r$ for $X / Y$ are determined. Next, we apply our results to a particular case of $X / Y$, when $X$ and $Y$ are two independent Hyper-Exponential distribution. Eventually, we apply our results to real life application in analyzing the performance of wireless communication systems and showing for some numerical values some related graphs.

\section{Some Preliminaries}

\subsection{The Hyper-Erlang distribution}

The Hyper-Erlang distribution is the mixture of $m$ mutually independent Erlang distributions or parallel $m$-phase Erlang distribution weighted with the probabilities $p_{i}$ and each $i^{\text {th }}$ Erlang stage $E_{i}$ has the shape parameter $k_{i}$ and rate parameter $\alpha_{i}$, for $1 \leq i \leq m$, written as $E_{\alpha_{i}, k_{i}} \sim E\left(\alpha_{i}, k_{i}\right)$. Let $X$ be a Hyper-Erlang distribution, then we write $X \sim H_{m}(\vec{p}, \vec{\alpha}, \vec{k})$, where $\vec{p}=\left(p_{1}, p_{2}, \ldots, p_{m}\right) \in[0,1]^{m}$ are the weighted probabilities with $\sum_{i=1}^{m} p_{i}=1$, $\vec{\alpha}=\left(\alpha_{1}, \alpha_{2}, \ldots, \alpha_{m}\right) \in \mathbb{R}_{+}^{m}$ are rate parameters, and $\vec{k}=\left(k_{1}, k_{2}, \ldots, k_{m}\right) \in \mathbb{N}^{m}$ are the shape parameters. The $\mathrm{PDF}$ of $X$ is given as

$$
f_{X}(t)=\sum_{i=1}^{m} p_{i} f_{E_{i}}(t)
$$

where $f_{E_{i}}(t)=\frac{\left(\alpha_{i} t\right)^{k_{i}-1} \alpha_{i} e^{-\alpha_{i} t}}{\left(k_{i}-1\right) !} I_{(0, \infty)}(t)$ is the PDF of the Erlang distribution $E_{\alpha_{i}, k_{i}} \sim E\left(\alpha_{i}, k_{i}\right)$. The cumulative distribution function of $X$ is $F_{X}(x)=\sum_{i=1}^{m} p_{i} F_{E_{i}}(x)=1-\sum_{i=1}^{m} \sum_{j=1}^{k_{i}-1} \frac{p_{i}\left(\alpha_{i} x\right)^{j} e^{-\alpha_{i} x}}{(j-1) !}$, the moment generating function is $\Phi_{X}(t)=\sum_{i=1}^{m} p_{i} \Phi_{E_{i}}(t)=\sum_{i=1}^{m} \frac{p_{i} \alpha_{i}^{m}}{\left(\alpha_{i}-t\right)^{m}}$, The moment of order $r$ is $E\left[X^{r}\right]=\sum_{i=1}^{m} p_{i} E\left[E_{\alpha_{i}, k_{i}}^{r}\right]=$ $\sum_{i=1}^{m} \frac{p_{i} \Gamma\left(r+k_{i}\right)}{\alpha_{i}^{r} \Gamma\left(k_{i}\right)}$. Then we have $E[X]=\sum_{i=1}^{m} \frac{p_{i} k_{i}}{\alpha_{i}}, E\left[X^{2}\right]=\sum_{i=1}^{m} \frac{p_{i} k_{i}\left(k_{i}+1\right)}{\alpha_{i}^{2}}$, see $[18,19,20]$.

The particular case of this distribution is when $\vec{k}=(1,1, \ldots, 1)$. This case is the Hyper-Exponential distribution where the $m$-phases are Exponential distribution, then $E_{\alpha_{i}, 1} \sim E\left(\alpha_{i}, 1\right)=\operatorname{Exp}\left(\alpha_{i}\right)$, the Exponential distribution with parameter $\alpha_{i}, 1 \leq i \leq m$. The PDF here is

$$
f_{X_{m}}(t)=\sum_{i=1}^{m} p_{i} \alpha_{i} e^{-\alpha_{i} t} I_{(0, \infty)}(t)
$$

This particular case can be written as $X \sim H_{m}(\vec{p}, \vec{\alpha}, \overrightarrow{1})=H_{m}(\vec{p}, \vec{\alpha})$.

\subsection{Generalized-F distribution}

Let $X$ be a random variable that has the Generalized-F distribution or called the generalized beta-prime distribution with three positive parameters $v_{1}, v_{2}$ and $\gamma$. The PDF of $\mathrm{X}$ is

$$
f\left(t, v_{1}, v_{2}, \gamma\right)=\frac{\gamma^{v_{2}} t^{v_{1}-1}}{B\left(v_{1}, v_{2}\right)(t+\gamma)^{v_{1}+v_{2}}}, t \geq 0
$$


where

$$
B\left(v_{1}, v_{2}\right)=\int_{0}^{1} t^{v_{1}-1}(1-t)^{v_{2}-1} d t
$$

is the usual Beta function, see [7]. Taking $\gamma=\frac{\beta}{\alpha}$, where $\alpha>0$ and $\beta>0$, we can write the PDF as

$$
f\left(t, v_{1}, v_{2}, \frac{\beta}{\alpha}\right)=\frac{\left(\frac{\beta}{\alpha}\right)^{v_{2}} t^{v_{1}-1}}{B\left(v_{1}, v_{2}\right)\left(t+\frac{\beta}{\alpha}\right)^{v_{1}+v_{2}}}
$$

The Generalized-F distribution is related to the $\mathrm{F}$ distribution with two parameters, where $\gamma X$ is a $\mathrm{F}$ distribution with $2 \alpha$ and $2 \beta$ degrees of freedom, [7] and [21]. Thus, the distribution of Generalized-F distribution can be obtained from this relation, see $[21,22,23]$. As a result we have the cumulative distribution function of $X$ is

$$
F(x)=I_{\frac{x}{x+\gamma}}\left(v_{1}, v_{2}\right),
$$

where $I_{x}(a, b)=\frac{B(x, a, b)}{B(a, b)}$ is the regularized incomplete beta function and $B(x, a, b)=\int_{0}^{x} t^{a-1}(1-t)^{b-1} d t$ is the incomplete beta function. The moment generating function of $X$ is

$$
\Phi(t)=\frac{\Gamma\left(v_{1}+v_{2}\right)}{\Gamma\left(v_{2}\right)} U\left(v_{1}, 1-v_{2},-\gamma t\right)
$$

where $U(a, b, z)=\frac{1}{\Gamma(a)} \int_{0}^{\infty} e^{-z t} t^{a-1}(1+t)^{b-a-1} d t$ is the confluent hypergeometric function of the second kind. Moment of order $k$ of $X$ is

$$
E\left[X^{k}\right]=\frac{\gamma^{k} \Gamma\left(v_{1}+k\right) \Gamma\left(v_{2}-k\right)}{\Gamma\left(v_{1}\right) \Gamma\left(v_{2}\right)} \text { when } k<v_{2}
$$

else diverges, thus the expectation is $E[X]=\frac{\gamma v_{2}}{\left(v_{2}-1\right)}$, when $v_{2}>1$ and $\operatorname{Var}[X]=\frac{\gamma^{2} 2 v_{1}\left(v_{1}+v_{2}-1\right)}{v_{2}^{2}\left(v_{2}-1\right)^{2}\left(v_{2}-2\right)}$ where $v_{2}>2$.

The particular case of the Generalized-F distribution is when $v_{1}=v_{2}=1$, which is the log-logistic distribution with parameter $1 / \gamma$ and 1 , we write

$$
X \sim G F(1,1, \gamma)=\log \operatorname{logistic}\left(\frac{1}{\gamma}, 1\right)
$$

when $v_{1}=v_{2}=1$. In this case we have the PDF of $X$ is

$$
f(t)=\frac{\gamma}{(t+\gamma)^{2}}
$$

if $t>0$ and $f(t)=0$ if $t \leq 0$, and $\mathrm{CDF}$ is

$$
F(x)=\frac{x}{x+\gamma}
$$

if $x>0$ and $f(x)=0$ if $x \leq 0$.

\section{Ratio of Hyper-Erlang Distribution}

In this section, we examine the ratio of the Hyper-Erlang distribution. A closed expression of the PDF is derived and written as a linear combination of the Generalized-F distribution. As a consequence, the expressions CDF, reliability function, hazard function, moment generating function and moments of order $r$ for this distribution are determined.

We suppose that $X$ and $Y$ are two independent Hyper-Erlang distribution. Then we take $X \sim$ $H_{m}(\vec{p}, \vec{\alpha}, \vec{k}), \vec{p}=\left(p_{1}, p_{2}, \ldots, p_{m}\right) \in[0,1]^{m}, \vec{\alpha}=\left(\alpha_{1}, \alpha_{2}, \ldots, \alpha_{m}\right) \in \mathbb{R}_{+}^{m}$, and $\vec{k}=\left(k_{1}, k_{2}, \ldots, k_{m}\right) \in \mathbb{N}^{m}$ and 
$Y \sim H_{n}(\vec{q}, \vec{\beta}, \vec{l}), \vec{q}=\left(q_{1}, q_{2}, \ldots, q_{n}\right) \in[0,1]^{n}, \vec{\beta}=\left(\beta_{1}, \beta_{2}, \ldots, \beta_{n}\right) \in \mathbb{R}_{+}^{n}$, and $\vec{l}=\left(l_{1}, l_{2}, \ldots, l_{n}\right) \in \mathbb{N}^{n}$. From Eq (1) the PDF of $X$ and $Y$ are

$$
f_{X}(t)=\sum_{i=1}^{m} p_{i} f_{E_{\alpha_{i}, k_{i}}}(t) \text { and } f_{Y}(t)=\sum_{j=1}^{n} q_{j} f_{E_{\beta_{j}, l_{j}}}(t)
$$

respectively, where $E_{\alpha_{i}, k_{i}} \sim E\left(\alpha_{i}, k_{i}\right), 1 \leq i \leq m$, and $E_{\beta_{j}, l_{j}} \sim E\left(\beta_{j}, l_{j}\right), 1 \leq j \leq n$.

Theorem 1

Let $X$ and $Y$ be two independent Hyper-Erlang distributed according to (11). Then the PDF of $X / Y$ is given by

$$
f_{X / Y}(t)=\sum_{i=1}^{m} \sum_{j=1}^{n} p_{i} q_{j} f_{W_{i, j}}(t)
$$

where $f_{W_{i, j}}(t)$ is the PDF of the Generalized-F distribution $W_{i, j} \sim G F\left(k_{i}, l_{j}, \frac{\beta_{j}}{\alpha_{i}}\right)$.

Proof

The PDF of ratio distribution $X / Y$ is given by $f_{X / Y}(t)=\int_{0}^{\infty} y f_{X}(y t) f_{Y}(y) d y$ for $t \geq 0$. However the PDF of $X$ and $Y$, are given in Eq (11), Thus we obtain that $f_{X / Y}(t)=\sum_{i=1}^{m} \sum_{j=1}^{n} p_{i} q_{j} \int_{0}^{\infty} y\left(f_{E_{\alpha_{i}, k_{i}}}(y t) f_{E_{\beta_{j}, l_{j}}}(y)\right) d y$. The product in this integral is a multiplication of the PDF of two Erlang distribution corresponding to their parameters which is given as $f_{E_{\alpha_{i}, k_{i}}}(y t) f_{E_{\beta_{j}, l_{j}}}(y)=\frac{\left(\frac{\beta_{j}}{\alpha_{i}}\right)^{l_{j}} t^{k_{i}-1}}{\left(k_{i}-1\right) !\left(l_{j}-1\right) !} y^{k_{i}+l_{j}-2} e^{-y\left(t+\frac{\beta_{j}}{\alpha_{i}}\right)}$ for $t \geq 0$ and $t<0$ gives $f_{Y / X}(t)=0$. Then, for $t \geq 0$,

$$
f_{X / Y}(t)=\sum_{i=1}^{m} \sum_{j=1}^{n} p_{i} q_{j} \frac{\left(\frac{\beta_{j}}{\alpha_{i}}\right)^{l_{j}} t^{k_{i}-1}}{\left(k_{i}-1\right) !\left(l_{j}-1\right) !} \int_{0}^{\infty} y^{k_{i}+l_{j}-1} e^{-y\left(t+\frac{\beta_{j}}{\alpha_{i}}\right)} d y
$$

To evaluate the above integral, we use the integral $\int_{0}^{\infty} x^{a} e^{-x b} d x=\frac{a !}{b^{a+1}}$ for $b>0$ and any positive integer $a$. Thus $\int_{0}^{\infty} y^{k_{i}+l_{j}-1} e^{-y\left(t+\frac{\beta_{j}}{\alpha_{i}}\right)} d y=\frac{\left(k_{i}+l_{j}-1\right) !}{\left(t+\frac{\beta_{j}}{\alpha_{i}}\right)^{k_{i}+l_{j}}}$. We obtain that

$$
f_{X / Y}(t)=\sum_{i=1}^{m} \sum_{j=1}^{n} p_{i} q_{j} \frac{\left(\frac{\beta_{j}}{\alpha_{i}}\right)^{l_{j}}\left(k_{i}+l_{j}-1\right) ! t^{k_{i}-1}}{\left(k_{i}-1\right) !\left(l_{j}-1\right) !\left(t+\frac{\beta_{j}}{\alpha_{i}}\right)^{k_{i}+l_{j}}}
$$

However, $\frac{\left(k_{i}-1\right) !\left(l_{j}-1\right) !}{\left(k_{i}+l_{j}-1\right) !}$ is the particular case of the beta function $B\left(k_{i}, l_{j}\right)$ in Eq (3), where $k_{i}$ and $l_{j}$ are integers. Therefore, we obtain that

$$
f_{X / Y}(t)=\sum_{i=1}^{m} \sum_{j=1}^{n} p_{i} q_{j} \frac{\left(\frac{\beta_{j}}{\alpha_{i}}\right)^{l_{j}} t^{k_{i}-1}}{B\left(k_{i}, l_{j}\right)\left(t+\frac{\beta_{j}}{\alpha_{i}}\right)^{k_{i}+l_{j}}}
$$

Referring to Eq (4), the expression in the above summation the PDF of the Generalized-F distribution, $G F\left(k_{i}, l_{j}, \frac{\beta_{j}}{\alpha_{i}}\right)$. Writing $W_{i, j} \sim G F\left(k_{i}, l_{j}, \frac{\beta_{j}}{\alpha_{i}}\right)$, we obtain the result.

Theorem 1 showed that the PDF of the ratio distribution of two independent Hyper-Erlang distribution is a linear combination of the Generalized-F distribution. From this linear form, we can find other related functions for $X / Y$, such as the $\mathrm{CDF}$, reliability function, hazard function, moment generating function and moment of order $r$. As a consequence of Theorem 1, we prove in the next corollaries that these related functions are also a linear combination of the those of the Generalized-F distribution. 


\section{Corollary 1}

Let $X$ and $Y$ be two independent Hyper-Erlang distributed according to (11). Then the CDF of $X / Y$ is

$$
F_{X / Y}(x)=\sum_{i=1}^{m} \sum_{j=1}^{n} p_{i} q_{j} I_{\frac{x}{x+\frac{\beta_{j}}{\alpha_{i}}}}\left(k_{i}, l_{j}\right)
$$

where $I_{x}(a, b)$ is the regularized incomplete beta function.

In the next corollaries, we give the expression of the reliability function and hazard function of $X / Y$.

\section{Corollary 2}

Let $X$ and $Y$ be two independent Hyper-Erlang distributed according to (11). Then the reliability function of $X / Y$ is

$$
R_{X / Y}(x)=\sum_{i=1}^{m} \sum_{j=1}^{n} p_{i} q_{j} I_{\frac{\gamma_{i j}}{x+\gamma_{i j}}}\left(k_{i}, l_{j}\right)
$$

and the hazard function of $X / Y$ is

$$
h_{X / Y}(t)=\frac{\sum_{i=1}^{m} \sum_{j=1}^{n} p_{i} q_{j} f_{W_{i, j}}(t)}{\sum_{i=1}^{m} \sum_{j=1}^{n} p_{i} q_{j} I_{\frac{\gamma_{i j}}{x+\gamma_{i j}}}\left(k_{i}, l_{j}\right)}
$$

where $I_{x}(a, b)$ is the regularized incomplete beta function and $\gamma_{i j}=\frac{\beta_{j}}{\alpha_{i}}, 1 \leq i \leq m$ and $1 \leq j \leq n$.

Proof

The reliability function of $X / Y$ is related to the CDF as $R_{X / Y}(x)=1-F_{X / Y}(x)$. We use the expression of $F_{X / Y}(x)$ in Corollary 1 as

$$
F_{X / Y}(x)=\sum_{i=1}^{m} \sum_{j=1}^{n} p_{i} q_{j} I_{\frac{x}{x+\frac{\beta_{j}}{\alpha_{i}}}}\left(k_{i}, l_{j}\right) .
$$

However, from [24], we have $I_{\frac{x}{x+\gamma_{i j}}}\left(k_{i}, l_{j}\right)=1-I_{1-\frac{x}{x+\gamma_{i j}}}\left(l_{j}, k_{i}\right)=1-I_{\frac{\gamma_{i j}}{x+\gamma_{i j}}}\left(l_{j}, k_{i}\right)$. Then we have

$$
F_{X / Y}(x)=\sum_{i=1}^{m} \sum_{j=1}^{n} p_{i} q_{j}-\sum_{i=1}^{m} \sum_{j=1}^{n} p_{i} q_{j} I_{\frac{\gamma_{i j}}{x+\gamma_{i j}}}\left(l_{j}, k_{i}\right)=1-\sum_{i=1}^{m} \sum_{j=1}^{n} p_{i} q_{j} I_{\frac{\gamma_{i j}}{x+\gamma_{i j}}}\left(k_{i}, l_{j}\right) .
$$

Thus, the reliability is $R_{X / Y}(x)=\sum_{i=1}^{m} \sum_{j=1}^{n} p_{i} q_{j} I_{\frac{\gamma_{i j}}{x+\gamma_{i j}}}\left(k_{i}, l_{j}\right)$. Moreover, the hazard function is $f_{X / Y}(t) / R_{X / Y}(t)$. Substituting the expressions of $f_{X / Y}(t)$ in Eq (12) and $R_{X / Y}(t)$,we obtain the result.

Corollary 3

Let $X$ and $Y$ be two independent Hyper-Erlang distributed according to (11). Then the moment generating function of $X / Y$ is

$$
\Phi_{X / Y}(t)=\sum_{i=1}^{m} \sum_{j=1}^{n} p_{i} q_{j} \Phi_{W_{i, j}}(t)
$$

where $\Phi_{W_{i, j}}(t)=\frac{\Gamma\left(k_{i}+l_{j}\right)}{\Gamma\left(l_{j}\right)} U\left(k_{i}, 1-l_{j},-\frac{\beta_{j} t}{\alpha_{i}}\right)$ and $U(a, b, z)$ is the confluent hypergeometric function of the second kind. 


\section{Proposition 1}

Let $X$ and $Y$ be two independent Hyper-Erlang distributed according to (11). Then the moment of $Z=X / Y$ of order $r$ is

$$
E\left[Z^{r}\right]=\sum_{i=1}^{m} \sum_{j=1}^{n} p_{i} q_{j} E\left[W_{i, j}^{r}\right]=\sum_{i=1}^{m} \sum_{j=1}^{n} \frac{p_{i} q_{j} \beta_{j}^{r} \Gamma\left(k_{i}+r\right) \Gamma\left(l_{j}-r\right)}{\alpha_{i}^{r} \Gamma\left(k_{i}\right) \Gamma\left(l_{j}\right)}
$$

for $r<\min \left\{l_{j}\right\}, 1 \leq j \leq n$ and $W_{i, j} \sim G F\left(k_{i}, l_{j}, \frac{\beta_{j}}{\alpha_{i}}\right)$.

Proof

From Eq (13) the moment generating function of $Z$ is

$$
\Phi_{Z}(t)=\sum_{i=1}^{m} \sum_{j=1}^{n} p_{i} q_{j} \Phi_{W_{i, j}}(t)
$$

and the $r^{t h}$ derivative is given as $\frac{d^{r} \Phi_{Z}(t)}{d t^{r}}=\sum_{i=1}^{m} \sum_{j=1}^{n} p_{i} q_{j} \frac{d^{r} \Phi_{W_{i, j}}(t)}{d t^{r}}$. Now, since the moment of order $r$ of $Z$ is the $r^{t h}$ derivative of $\Phi_{Z}(t)$ at $t=0$, this gives that $E\left[Z^{r}\right]=\sum_{i=1}^{m} \sum_{j=1}^{n} p_{i} q_{j} E\left[W_{i, j}^{r}\right]$, where $E\left[W_{i, j}^{r}\right]$ is the moment of order $r$ of the Generalized-F distribution $W_{i, j} \sim G F\left(k_{i}, l_{j}, \frac{\beta_{j}}{\alpha_{i}}\right)$.

On the other hand, Eq (7) gives that

$$
E\left[W_{i, j}^{r}\right]=\frac{\beta_{j}^{r} \Gamma\left(k_{i}+r\right) \Gamma\left(l_{j}-r\right)}{\alpha_{i}^{r} \Gamma\left(k_{i}\right) \Gamma\left(l_{j}\right)}
$$

when $r<\min \left\{l_{j}\right\}, 1 \leq j \leq n$. Thus, we obtain the required results.

From proposition 1, we conclude some particular moments. The expectation, for $r=1, E[Z]=\sum_{i=1}^{m} \sum_{j=1}^{n} \frac{p_{i} q_{j} \beta_{j} k_{i}}{\alpha_{i}\left(l_{j}-1\right)}$ for $l_{j} \neq 1,1 \leq j \leq n$, also for $r=2, E\left[Z^{2}\right]=\sum_{i=1}^{m} \sum_{j=1}^{n} \frac{p_{i} q_{j} \beta_{j}^{2} k_{i}\left(k_{i}-1\right)}{\alpha_{i}^{2}\left(l_{j}-1\right)\left(l_{j}-2\right)}$ for $l_{j} \neq 1,2$ and $1 \leq j \leq n$.

\section{Ratio of Hyper-Exponential Distribution}

Next, we consider the particular case of the Hyper-Erlang distribution, the Hyper-Exponential distribution. Let $X$ and $Y$ be two independent Hyper-Exponential random variables each distribution of different stages. Thus, $X \sim$ $H_{m}(\vec{p}, \vec{\alpha}), \vec{p}=\left(p_{1}, p_{2}, \ldots, p_{m}\right) \in[0,1]^{m}, \sum_{i=1}^{m} p_{i}=1$ and $\vec{\alpha}=\left(\alpha_{1}, \alpha_{2}, \ldots, \alpha_{m}\right) \in \mathbb{R}_{+}^{m}$, and $Y \sim H_{n}(\vec{q}, \vec{\beta})$, $\vec{q}=\left(q_{1}, q_{2}, \ldots, q_{n}\right) \in \mathbb{R}_{+}^{m}, \sum_{j=1}^{n} q_{j}=1$, and $\vec{\beta}=\left(\beta_{1}, \beta_{2}, \ldots, \beta_{n}\right) \in[0,1]^{m}$. Then from Eq (2) the PDF of $X$ and $Y$ are

$$
f_{X}(t)=\sum_{i=1}^{m} p_{i} \alpha_{i} e^{-\alpha_{i} t} \text { and } f_{Y}(t)=\sum_{j=1}^{n} q_{j} \beta_{j} e^{-\beta_{j} t}
$$

We note again that in this case $X \sim H_{m}(\vec{p}, \vec{\alpha}, \vec{k})=H_{m}(\vec{p}, \vec{\alpha})$ where $\vec{k}=(1,1, \ldots, 1)$ and $Y \sim$ $H_{n}(\vec{q}, \vec{\beta}, \vec{l})=H_{n}(\underline{\vec{q}}, \vec{\beta})$ where $\vec{l}=(1,1, \ldots, 1)$. Then referring to our results in section 3 and by taking the particular cases when $\vec{k}=(1,1, \ldots, 1)$ and $\vec{l}=(1,1, \ldots, 1)$, we obtain the results of the ratio of two independent Hyper-Exponential distribution. We state them in the following corollaries.

\section{Corollary 4}

Let $X \sim H_{m}(\vec{p}, \vec{\alpha})$ and $Y \sim H_{n}(\vec{q}, \vec{\beta})$ be two independent Hyper-Exponential distribution. Then the PDF of $X / Y$ is

$$
f_{X / Y}(t)=\sum_{i=1}^{m} \sum_{j=1}^{n} \frac{p_{i} q_{j} \frac{\beta_{p}}{\alpha_{i}}}{\left(t+\frac{\beta_{p}}{\alpha_{i}}\right)^{2}}
$$


for $t>0$ and $f_{X / Y}(t)=0$, for $t \leq 0$. The CDF is

$$
F_{X / Y}(x)=\sum_{i=1}^{m} \sum_{j=1}^{n} \frac{p_{i} q_{j} x}{x+\frac{\beta_{j}}{\alpha_{i}}}
$$

for $x>0$ and $F_{X / Y}(x)=0$, for $x \leq 0$. The reliability function is

$$
R_{X / Y}(t)=\sum_{i=1}^{m} \sum_{j=1}^{n} \frac{p_{i} q_{j}\left(\frac{\beta_{j}}{\alpha_{i}}\right)}{t+\frac{\beta_{j}}{\alpha_{i}}}
$$

for $t>0$ and $R_{X / Y}(t)=0$, for $t \leq 0$. The hazard function is

$$
h_{X / Y}(t)=\frac{\sum_{i=1}^{m} \sum_{j=1}^{n} \frac{p_{i} q_{j} \frac{\beta_{j}}{\alpha_{i}}}{\left(t+\frac{\beta_{p}}{\alpha_{i}}\right)^{2}}}{\sum_{i=1}^{m} \sum_{j=1}^{n} \frac{p_{i} q_{j}\left(\frac{\beta_{j}}{\alpha_{i}}\right)}{t+\frac{\beta_{j}}{\alpha_{i}}}}
$$

for $t>0$ and $h_{X / Y}(t)=0$, for $t \leq 0$.

Proof

The proof is directly obtained by taking the particular case of the ratio of two independent Hyper-Erlang distribution given in Theorem 1 and Corollaries 1 and 2. In this particular case we have from Equation (8), $W_{i, j} \sim G F\left(1,1, \frac{\beta_{j}}{\alpha_{i}}\right)=\log \operatorname{logistic}\left(\frac{\alpha_{i}}{\beta_{j}}, 1\right)$ and knowing that PDF and CDF of $W_{i, j}$ are given in Eq (9) and Eq (10) as $f(t)=\frac{\frac{\beta_{j}}{\alpha_{i}}}{\left(t+\frac{\beta_{j}}{\alpha_{i}}\right)^{2}}$ and $F(x)=\frac{x}{x+\frac{\beta_{j}}{\alpha_{i}}}$ respectively. Thus, the results are obtained.

\section{Application}

In this section we will apply our result to real life application in analyzing the performance of wireless communication systems. Ratios of random variables have been widely utilized in reporting results in the biological, physical and social sciences. Examples include Mendelian inheritance ratios in genetics, mass to energy ratios in nuclear physics, target to control precipitation in meteorology and inventory ratios in economics. The distributions of ratios of random variables are also of interest in performance analysis of wireless communication systems. Radio wave propagation through wireless channels is a complicated phenomenon characterized by fading which is the result of multipath propagation due to reflection, refraction and scattering of radio waves by buildings and other structures. When a received signal experiences fading during transmission, its envelope fluctuates over time. Desired signal in wireless communication systems is also subjected to cochannel interference $(\mathrm{CCI})$ due to reuse of radio frequencies which is essential in increasing system capacity. Multi-hop relaying communication has a number of advantages over traditional direct-link transmission in the areas of connectivity, deployment, power saving and channel capacity. Relaying techniques enable network connectivity where traditional architectures are impractical due to location constraints. Multi-hop networks technology is a promising solution for future cellular, wireless local area networks (WLANs) and hybrid networks. It is reason why dual and multi-hop wireless communication systems operating in fading channels have been an important field of research in the past few years. Motivated by the preceding, we will find the closed form, using our methodology, of the probability distribution of the ratio $X / Y$ in multi-hop relaying communication (figure 1,2 ) system. Where $X$ represents signal envelope which suffers from fading, while $Y$ represents envelope of CCI simultaneously affected by both fading and shadowing. Therefore, random variable $X / Y$ presents signal-to-interference ratio (SIR), hence its probability distribution can model different transmission environments. $X$ and $Y$ are two Hyper-Erlang random 


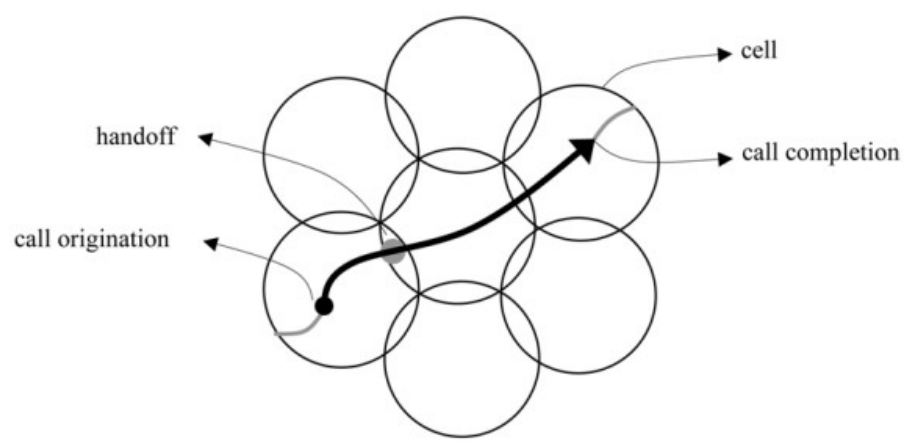

Figure 1. Wireless communication systems

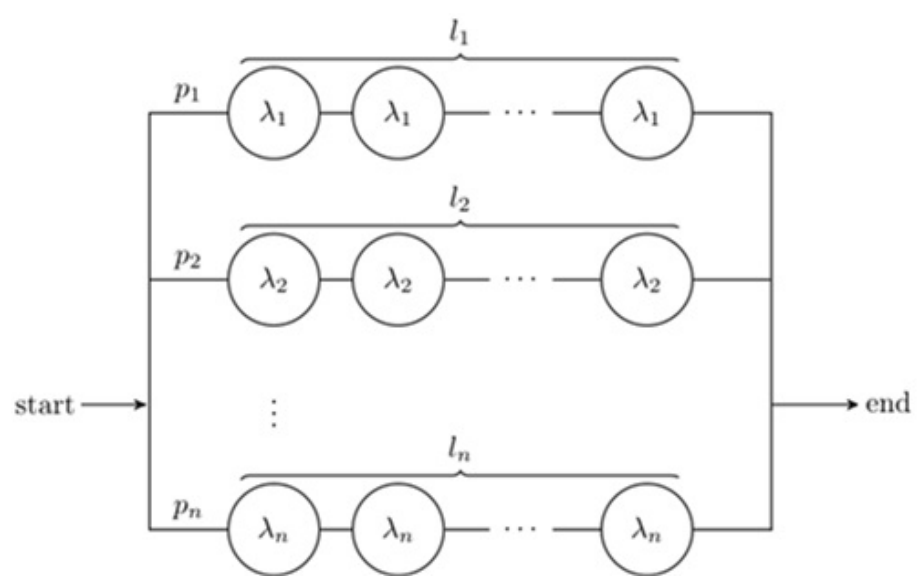

Figure 2. Multi-hop networks with Hyper-Erlang distribution

variables.

Numerical values: Let $X$ and $Y$ be two independent Hyper-Erlang distribution with $X \sim H_{3}(\vec{p}, \vec{\alpha}, \vec{k}), \vec{p}=$ $(0.5,0.2,0.3), \vec{\alpha}=(1,3,5)$, and $\vec{k}=(2,4,3)$ and $Y \sim H_{4}(\vec{q}, \vec{\beta}, \vec{l}), \vec{q}=(0.2,0.1,0.5,0.2), \vec{\beta}=(1,4,6,2)$, and $\vec{l}=(3,5,2,3)$. By applying Theorem 1 , we obtain that the PDF of $X / Y$ is

$$
\begin{aligned}
f_{X / Y}(t)= & \frac{1.2 t}{(1+t)^{5}}+\frac{9.6 t}{(2+t)^{5}}+\frac{1536 t}{(4+t)^{7}}+\frac{54 t}{(6+t)^{4}}+\frac{194.4 t^{3}}{(1+3 t)^{7}}+\frac{1555.2 t^{3}}{(2+3 t)^{7}}+\frac{464486 t^{3}}{(4+3 t)^{9}} \\
& +\frac{5832 t^{3}}{(6+3 t)^{6}}+\frac{225 t^{2}}{(1+5 t)^{6}}+\frac{1800 t^{2}}{(2+5 t)^{6}}+\frac{403200 t^{2}}{(4+5 t)^{8}}+\frac{8100 t^{2}}{(6+5 t)^{5}}
\end{aligned}
$$

for $t>0$ and $f_{X / Y}(t)=0$ for $t \leq 0$. Figure 3 shows the curve of $f_{X / Y}(t)$ and Figures 4,5 and 6 show the CDF, reliability and hazard function of $X / Y$ respectively, obtained in Corollaries 1 and 2.

\section{Conclusion}

The closed expressions of the PDF, CDF, reliability function, hazard function, moment generating function and the $r^{t h}$ moment of the ratio of two independent Hyper-Erlang distribution are given. The expressions are proved to be a linear combination of the Generalized-F distribution. Next, we observe the particular case, the ratio of two 


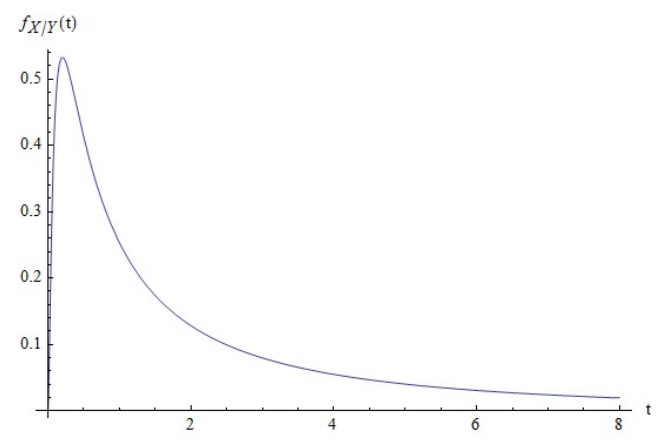

Figure 3. PDF

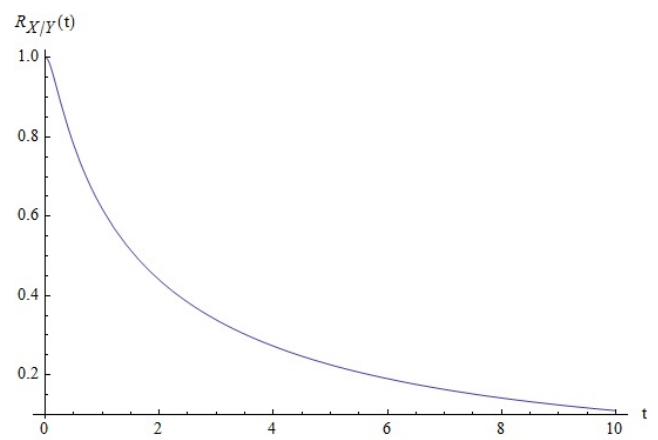

Figure 5. Reliability Function

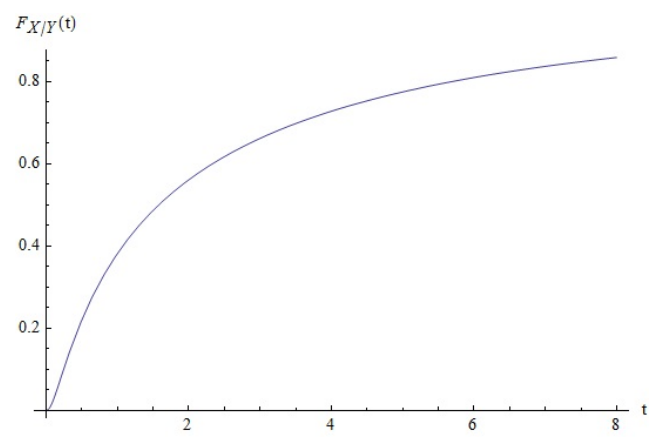

Figure 4. CDF

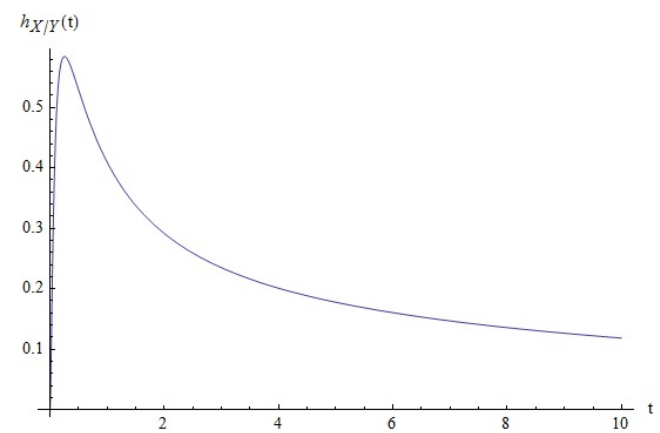

Figure 6. Hazard Function

independent Hyper-Exponential distribution and expressions are again established in a particular and more simple form. Eventually, we illustrate our results in an example, by finding the ratio distribution and showing some graphs.

\section{REFERENCES}

1. M. Masoom Ali, M. Pal, and J. Woo, On the Ratio of Inverted Gamma Variates, Austrian Journal of Statistic,vol. 36, no. 2, pp. $153-15,2007$..

2. L. Idrizi, On The Product and Ratio of Pareto and Kumarazwamy Random Variables, Mathematical Theory and Modeling, vol. 4, no. 3, 2014.

3. M. Shakil and B. M. Golam Kibria, Exact Distribution of the Ratio of Gamma and Rayleigh Random Variables, Pakistan Journal of Statistics and Operation Research, vol. 2, no. 2, pp. 87-98, July 2006.

4. S. Nadarajah and D. Choi, Arnold and Strauss's bivariate exponential distribution products and ratios, New Zealand Journal of Mathematics, vol. 35, pp. 189-1999, 2006.

5. S. Park, On the Distribution Functions of Ratios Involving Gaussian Random Variables, ETRI Journal, vol. 32, no. 6, December 2010.

6. S. Nadarajah and S. Kotz, On the product and ratio of $t$ and Bessel random variables, Bulletin of the Institute of Mathematics Academia Sinica (New Series), vol. 2, no. 1, pp. 55-66, 2007.

7. T. Pham-Gia and N. Turkkan, Operations on the Generalized F-Variables, and Applications, A Journal of Theoretical and Applied Statistics, vol. 36, no. 3, pp. 195-209, 2002. doi:10.1080/02331880212855.

8. G. Marsaglia, Ratios of Normal Variables and Ratios of Sums of Uniform Variables, Journal of the American Statistical Association. vol. 60, no. 309, pp. 193-204, March 1965.

9. P. J. Korhonen and S. C. Narula, The Probability Distribution of the Ratio of the Absolute Values of Two Normal Variables, Journal of Statistical Computation and Simulation vol. 33, no. 3, pp. 173-182,1989. doi:10.1080/00949658908811195.

10. S. J. Press, The t-ratio distribution, Journal of the American Statistical Association, vol. 64, no. 325, pp. 242-252, 1969.

11. A. P. Basu and R. H. Lochner, On the distribution of the ratio of two random variables having generalized life distributions, Technometrics, vol. 13, no. 2, pp. 281-287, 1971.

12. D. L. Hawkins and C. P. Han, Bivariate distributions of some ratios of independent noncentral chi-square random variables, Communications in Statistics - Theory and Methods, vol. 15, no. 1, pp. 261-277,1986. doi:10.1080/03610928608829120.

13. S. B. Provost, On the distribution of the ratio of powers of sums of gamma random variables, Pakistan Journal Statistic, vol. 5, no. 2, pp. 157-174, 1989. 
14. T. Pham-Gia, Distributions of the ratios of independent beta variables and applications, Communications in Statistics - Theory and Methods, vol. 29, no. 12, pp. 2693-2715, 2000. doi:10.1080/03610920008832632

15. S. Nadarajaha, and A.K. Gupta, On the ratio of logistic random variables, Computational Statistics \& Data Analysis, vol. 50, pp. 1206-1219, 2006.

16. S. Nadarajaha, S. Kotz, On the Ratio of Frèchet Random Variables, Quality \& Quantity 40, pp. 861-868, 2006.

17. S. Nadarajah, The linear combination, product and ratio of Laplace random variables. Statistics, A Journal of Theoretical and Applied Statistics, vol. 41, no. 6, pp. 535-545, 2007.

18. A. Thümmler, P. Buchholz, and M. Telek, A novel approach for phase-type fitting with the EM algorithm, IEEE Trans. Dependable Sec. Comput., Vol. 3, No. 3, pp 245-258, 2006.

19. A. V. Pechinkin, C. D’Apice, P. P. Bocharov, S. Salerno, Queueing Theory, De Gruyter Incorporated, Walter, Modern Probability and Statistics Ser, 2003.

20. Y. Fang, Hyper-Erlang Distribution Model and Its Application in Wireless Mobile Networks, Wireless Networks, vol. 7, pp. 211-219, 2001.

21. T. Pham-Gia and N. Turkkan, Distributions of Ratios: From Random Variables to Random Matrices, Open Journal of Statistics, vol. 1, no. 2, pp. 93-104, 2011. doi: 10.4236/ojs.2011.12011.

22. T. Pham-Gia and Q. P. Duong, The generalized beta and F-distributions in statistical modelling, Mathematical and Computer Modelling, vol. 12, no. 12, pp. 1613-1625, 1989.

23. N. Johnson, S. Kotz, and N. Balakhrishnan, Continuous Univariate Distributions, John Wiley and Sons, New York, vol. 2, 2nd Edition, 1995.

24. W. Press, S. Teukolsky, W. Vetterling, B. Flannery and M. Metcalf, Numerical Recipes in C: The Art of Scientific Computing, Cambridge University Press, Third Edition, 2007. 